\title{
Experimental studies into the performance of the lead coolant axial pump wet ends to justify main circulation pumps for the HMLC reactor plant circuits*
}

\author{
Aleksandr V. Beznosov ${ }^{1}$, Pavel A. Bokov ${ }^{1}$, Aleksandr V. Lvov ${ }^{1}$, Tatyana A. Bokova ${ }^{1}$, \\ Nikita S. Volkov ${ }^{1}$, Aleksandr R. Marov ${ }^{1}$ \\ 1 Nizhny Novgorod State Technical University n.a. R. Ye. Alekseyev, 24 Minina St., Nizhny Novgorod, 603950, Russia \\ Corresponding author: Tatyana A. Bokova (tatabo@bk.ru)
}

Academic editor: Boris Balakin • Received 25 September 2019 • Accepted 13 July 2020 • Published 11 September 2020

Citation: Beznosov AV, Bokov PA, Lvov AV, Bokova TA, Volkov NS, Marov AR (2020) Experimental studies into the performance of the lead coolant axial pump wet ends to justify main circulation pumps for the HMLC reactor plant circuits. Nuclear Energy and Technology 6(3): 143-147. https://doi.org/10.3897/nucet.6.57736

\begin{abstract}
The paper presents the results of the studies to justify the design solutions for the main circulation pumps of the heavy liquid-metal cooled reactor plant circuits. A substantial difference has been shown in the performance of pumps for the heavy liquid-metal coolant transfer. The studies have confirmed the qualitative difference in the cavitation performance of coolants, the state of the gases and vapors they contain, the influence of supply and discharge devices, and the effects of the impeller blade section performance and geometry and the hub-tip ratio on the pump performance. The studies were performed based on NNSTU's lead-cooled test facilities with the coolant temperature in a range of 440 to $550{ }^{\circ} \mathrm{C}$ and the coolant flow rate of up to $2000 \mathrm{t} / \mathrm{h}$. The outer diameter of the impellers and the straightening devices was about $200 \mathrm{~mm}$, and the thickness of the flat $08 \mathrm{Kh} 18 \mathrm{~N} 10 \mathrm{~T}$-steel blades was $4.0 \mathrm{~mm}$ and that of the airfoil blades was up to $6.0 \mathrm{~mm}$. The pump shaft speed changed in a stepped manner from $600 \mathrm{rpm}$ to $1100 \mathrm{rpm}$ after each $100 \mathrm{rpm}$.

The studies were conducted to justify the engineering and design solutions for pumps as applied to conditions of small and medium plants with fast neutron lead cooled reactors currently under investigation at NNSTU (BRS-GPG). The experimental results can be recommended for use to design other HLMC transfer pumps.
\end{abstract}

\section{Keywords}

Fast neutron reactor, main circulation pump, heavy liquid-metal coolant, impellers, impeller blades, pump supply and discharge

\section{Introduction}

There is no currently experience of designing and operating pumps to transfer heavy liquid-metal coolant $(\mathrm{Pb}$, $\mathrm{Pb}-\mathrm{Bi}$ eutectics) (HLMC) as applied to reactor plants (Be- znosov et al. 2006). Along with employing traditional methods of dynamic pump design (Karelin 1975, Lomakin 1966, Budov 1986, Mikhaylov and Malyushenko 1977, Rozhdestvenskiy 1977), an integrated experimental study is conducted at NNSTU to determine the specific nature of

* Russian text published: Izvestiya vuzov. Yadernaya Energetika (ISSN 0204-3327), 2020, n. 2, pp. 64-72. 
the axial pump wet end operation in HLMC (Chechyotkin 1971) as applicable to the conditions of lead and lead-bismuth cooled reactor circuits (Beznosov and Bokova 2012, Beznosov et al. 2009, 2016, Dragunov et al. 2015).

Cavitation behavior of HLMC in the considered conditions was explored and determined at the initial stage. The absence of conventional vaporous cavitation in the reactor circuit conditions was experimentally confirmed using independent methods. The conditions for its occurrence, development, and progression have been determined (Bokov 2015). The state of the gas and gas-vapor mixtures in HLMC and their effects on the operation of the blade pump wet ends have been studied (Bokov 2015). Further studies were conducted as applied to the conditions of the BRS GPG reactor facility under investigation at NNSTU (Beznosov et al. 2018). Dependences of the axial pump performance (head, delivery rate, efficiency) on the parameters of the pump wet end structural components were studied experimentally. Based on results of an experimental data analysis, the wet end parameters were selected with which the pump had the maximum performance (head, delivery rate, efficiency) of the tested parameters, and wet ends were manufactured with such flat-blade impeller parameters. A wet end with improved parameters of its components was studied experimentally. The performance of this (head, delivery rate, efficiency) was compared with the performance of wet ends with flat and airfoil pump impeller blades. Further stages are expected to involve endurance tests of the updated improved design, and then the impeller blade geometry airfoil design and testing.

\section{Experimental procedures and key results}

The experimental studies were performed as applied to the conditions of small plants with fast neutron lead and lead-bismuth cooled reactors with horizontal steam generators (BRS-GPG) under investigation at NNSTU (Beznosov et al. 2018). Experiments were performed based on NNSTU's lead cooled test benches at temperatures of 440 to $550{ }^{\circ} \mathrm{C}$. The wet end components, including the blades and the impellers (flat and airfoil types) of 4.0 to $6.0 \mathrm{~mm}$, were made of $08 \mathrm{Kh} 18 \mathrm{~N} 10 \mathrm{~T}$ steel. The external diameter of the impeller and straightening device blades (except as otherwise specified) was about $200 \mathrm{~mm}$, and the impeller sleeve diameter was $82 \mathrm{~mm}$ (Fig. 1). The shaft speed of the NSO-01 NGTU pump with 3 to 8 fixed impeller blades and of the NSO-02 NGTU pump with 4 rotating blades was changed in steps of $100 \mathrm{rpm}$ in a range of 600 to 1100 $\mathrm{rpm}$. The lead coolant flow rate in the circulation circuit, with the circulation line control valve fully opened, reached $2000 \mathrm{t} / \mathrm{h}$ with the shaft speed being $1100 \mathrm{rpm}$.

Except as otherwise specified (with beryllium and aluminum based coating formation), protective coatings were formed on the surfaces of the pump wet end struc-

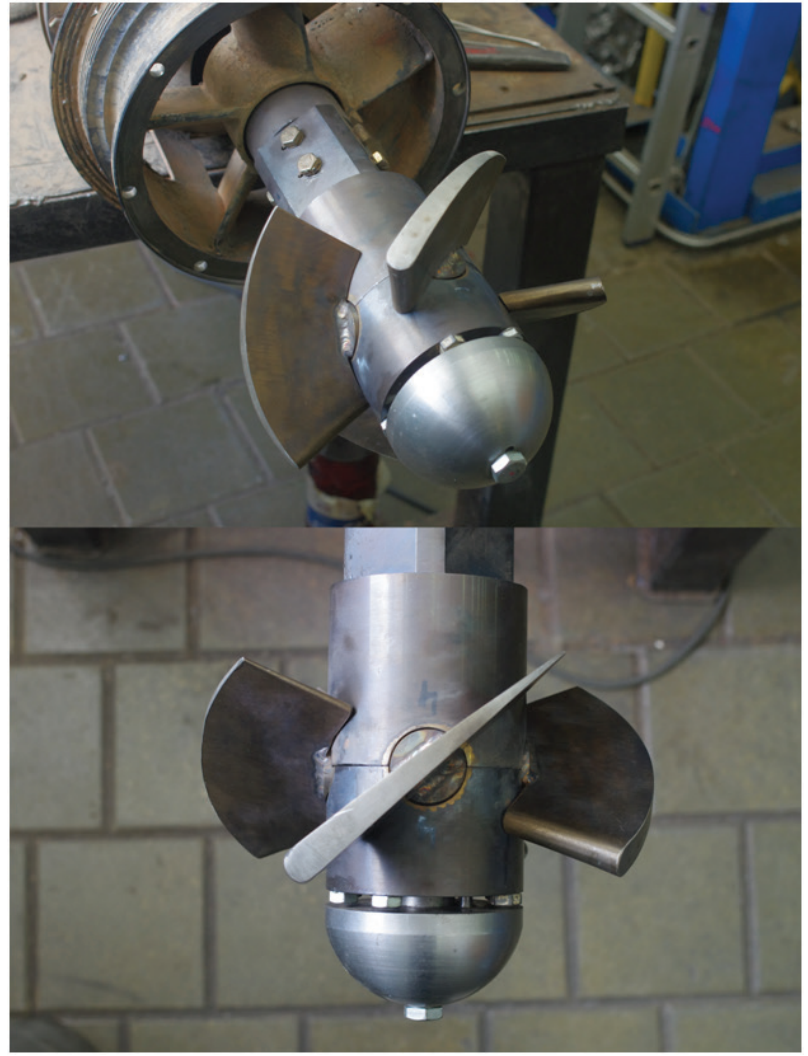

Figure 1. NSO-02 NGTU pump rotating assembly.

tural materials and maintained by oxygen control in the test bench lead coolant thanks to which the concentration of the thermodynamically active oxygen in lead was kept in a range of $10^{-5}$ to $10^{\circ}$.

The initial stage in the exploratory test series consisted in determining experimentally the dependences of the test bench axial pump performance (head, delivery rate, efficiency) on the scalable wet end structural components, the composition and geometry of which were specified in the test procedure program.

The following experiments were conducted at stage 1 .

1. Experimental studies into the dependences of the pump performance on the parameters of the straightening devices installed at the impeller flow outlet and inlet (Beznosov et al. 2019, 2019a). For the three straightening device design modifications installed downstream of the impellers, the blade space flow inlets had the angles of 22,24 , and $28^{\circ}$, and the number of the straightening device blades was five. The results of the studies for the straightening device designs at the impeller outlets and inlets have shown that both the pump delivery rate and head, other things being equal, do not change in principle. The findings have proved to be unexpected and differing greatly from the known water transfer pump solutions. The obtained result may be explained by a much greater degree of the wet end reactivity as compared with that expected. Additional tests performed with the straightening de- 
vices having been dismantled showed a much better pump performance as compared with the case of these being in place.

2. Experimental studies into the effects of the impeller inlet flow spin relative to the pump impeller rotating direction (Beznosov et al. 2019). The studies were conducted with changeable parts mounted at the pump inlet with the deflection angles of the four blades being $-18^{\circ}, 0^{\circ}$, and $+18^{\circ}$. It has been shown that the pump delivery rate is conservative as against the test results. For all spin device designs, the pump head is approximately equal and is notably smaller as compared with the pump head with the flow spin devices removed.

3. Experimental studies into the dependences of the pump performance (head, delivery rate, efficiency) on the impeller cascade parameters (Beznosov et al. 2014, 2014a, 2017). The effects of the key cascade parameters were studied, including, as shown in (Karelin 1975, Lomakin 1966, Budov 1986, Mikhaylov and Malyushenko 1977, Rozhdestvenskiy 1977, Papyr 1970), the following: $\alpha$ is the cascade blade incidence; $Z$ is the number of the impeller blades; $l / t$ is the cascade solidity. For the studies, the number of the blades mounted successively to the impeller was $3,4,6$, and 8 ; and the incidence for each set of blades was $9,15,22,32,38,41$, and $46^{\circ}$. The variable cascade solidity ( 0.8 to 1.2$)$ was achieved by changing the blade chord length with changing the area of each blade. The studies were conducted with three positions of the control gate valve wedge while changing respectively the coolant circulation line drag. A major dependence of the pump performance on the blade incidence has been recorded with bending points observed. The pump performance is less dependent on the number of the blades and the cascade solidity (Beznosov et al. 2019a).

4. Experimental studies into the dependences of the pump performance on the cascade blade inlet bend geometry (Beznosov et al. 2019). Impellers of three designs with four flat (non-airfoil) blades of a plate of $\delta=4.0 \mathrm{~mm}$ were mounted successively to the pump shaft for the studies. In all of the designs, each blade had a bend in the direction of the blade incidence decrease on the front one third of the blade chord stream-wise: by $\beta=4^{\circ}\left(\alpha-4^{\circ}\right)$ for design a), and by $\beta=8^{\circ}\left(\alpha-8^{\circ}\right)$ for design $\left.b\right)$; there were no bend $\left(\beta=8^{\circ}\right)$ for design $\left.\mathrm{c}\right)$. The blade chords remained straight. The impeller blade incidence, $\alpha$, was $16,20,24$, and $28^{\circ}$ for each blade bend option $\left(\beta=4^{\circ}, \beta=8^{\circ}, \beta=0^{\circ}\right)$; and the shaft speed was $n=600,700,800,900,1000$, and $1100 \mathrm{rpm}$. The experiments showed a major change in the pump performance depending on the bending angle of the chord's front one third: the difference reached $20 \%$ with one and the same blade incidence (Beznosov et al. 2019). The tests made it possible to choose the best design for the next test stage.
5. Experimental studies into the dependences of the pump performance on the impeller cascade blade outlet geometry (Beznosov et al. 2019a). The impellers were mounted to the pump shaft successively with the blade incidences of 16,20 , and $28^{\circ}$. For each blade incidence, the bending angle for one third of the blade outlet was $-8,-4,0,+4$, and $+8^{\circ}$ relative to the straight flat blade. The experiments showed a major dependence of the pump performance on the bending angle for one third of the blade outlet, this difference reaching 15 to $20 \%$. The tests made it possible to choose the best design for the next test stage.

6. Experimental studies into the dependences of the pump performance on the impeller's hub-tip ratio. The performance of the pumps with an external impeller blade diameter of $\varnothing 213 \mathrm{~mm}$ and with four sleeves of the diameter $\varnothing 82, \varnothing 96,5, \varnothing 109$, and $\varnothing 120 \mathrm{~mm}$ with flat impeller blades $(\delta=4.0 \mathrm{~mm})$ were studied in the process of the experiments. There was a minor smooth decrease in the pump performance recorded as the sleeve diameter increased (Beznosov et al. 2019), which somewhat disagrees with the recommendations in the references below.

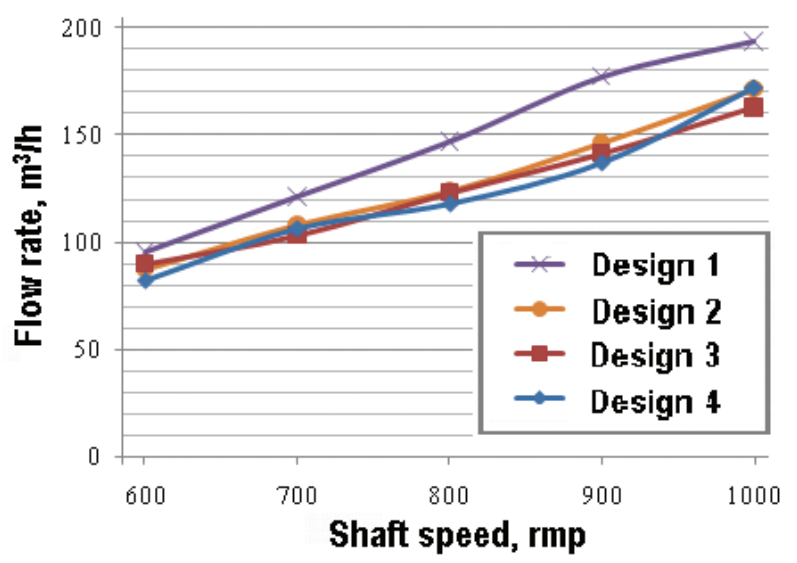

Figure 2. Lead coolant pump delivery rate as a function of speed, $T=450{ }^{\circ} \mathrm{C}$.

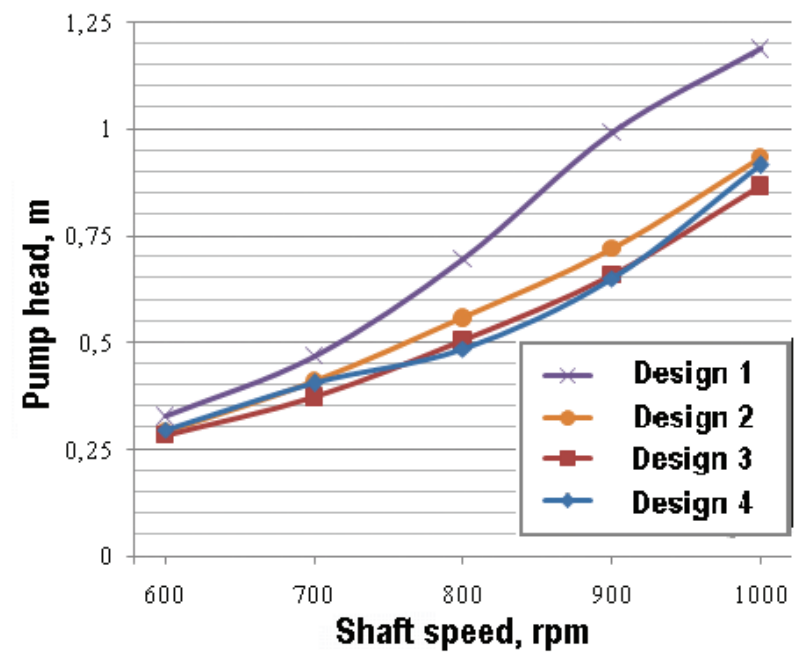

Figure 3. Pump head as a function of shaft speed, $T=450{ }^{\circ} \mathrm{C}$. 


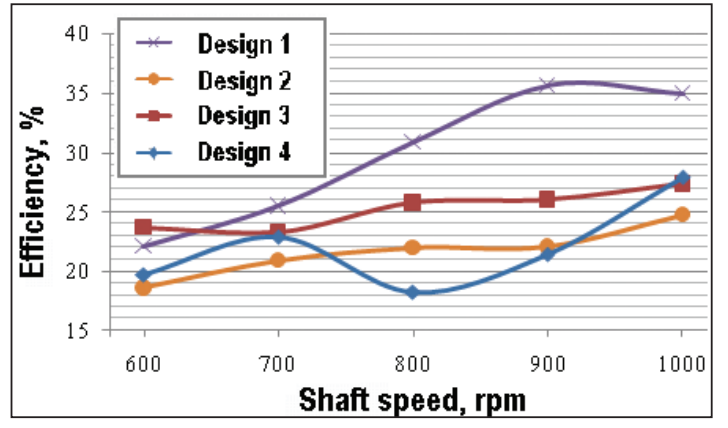

Figure 4. Pump efficiency as a function of shaft speed, $T=$ $450{ }^{\circ} \mathrm{C}$.

The next investigation stage involved a comparative analysis of the obtained results. The wet end components were chosen based on the analysis results, with which the pumps had the maximum performance (head, delivery rate, efficiency), and manufactured with such flat-blade impeller parameters. The results of testing the optimized pump wet end design have shown the following.

As shown by the results of the test's stage 1 (Beznosov et al. 2019), the wet end design with optimized components provides for a much better pump performance as compared with all of the designs tested earlier, including (Figs 2-4).

a) the flat-blade designs with the flat blade incidence of $\alpha=20^{\circ}$, the blade inlet bending angle of $4^{\circ}$, and the outlet bending angle of $0^{\circ}$ (curve 2 );

b) the flat-blade design with the flat blade incidence of $\alpha=20^{\circ}$, the inlet bending angle of $0^{\circ}$, and the outlet bending angle of $8^{\circ}$ (curve 3 );

c) the best blade system design of those tested earlier, with aluminum-based protective coatings and airfoil blades (curve 4).

\section{References}

- Beznosov AV, Antonenkov MA, Bokov PA, Baranova VS, Kustov MS (2009) Specific features of circulation pumps in lead and lead-bismuth cooled reactor circuits. Izvestiya vysshykh uchebnykh zavedeniy. Yadernaya energetika 4: 155-160. [in Russian]

- Beznosov AV, Bokov PA, Bokova TA (2016) Technologies and Main Equipment of Circuits in Reactor Facilities, Commercial and Research Test Benches with Lead and Lead-Bismuth Coolants. NGTU Publ., Nizhny Novgorod, 488 pp. [in Russian]

- Beznosov AV, Bokov PA, Zudin AD, Lvov AV, Bokova TA, Trushkov NV (2014) Hydrodynamics and efficiency of models of MCP BREST-OD-300 in a lead coolant at the FT-4 stand of the NNSTU. Trudy NGTU im. R.E. Alekseeva S5: 370. [in Russian]

- Beznosov AV, Bokova TA (2012) Equipment of Power Circuits with Heavy Liquid Metal Coolants in Nuclear Power Engineering. NGTU Publ., Nizhny Novgorod, 536 pp. [in Russian]

- Beznosov AV, Bokova TA, Bokov PA, Volkov NS, Karbyshev AA (2018) Specific solutions of the reactor circuit of the BRS-GPG

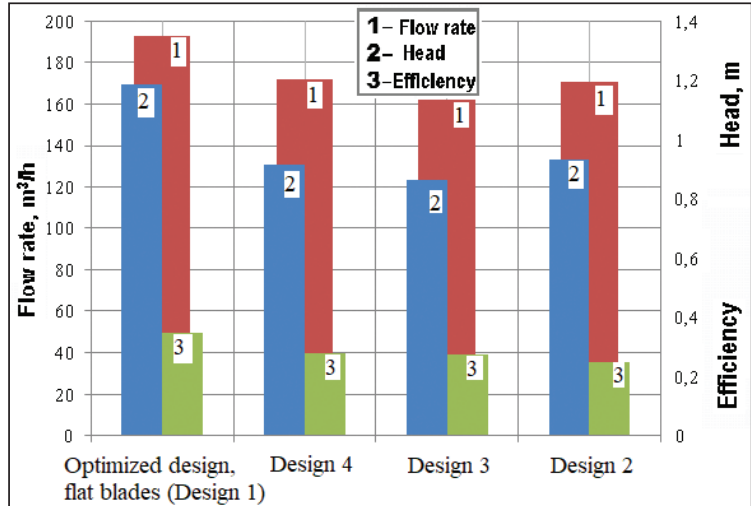

Figure 5. Performance of the pump wet end designs $\left(T=450^{\circ} \mathrm{C}\right.$, $n=1000 \mathrm{rpm})$.

The pump delivery rate in the event of the optimized design was 10 to $15 \%$ excessive (Fig. 2), the pump shaft speed being $1000 \mathrm{rpm}$. The pump head in the optimized wet end design was 20 to $25 \%$ excessive (see Fig. 3 ) with the pump shaft speed of $1000 \mathrm{rpm}$. The optimized wet end design also had a greater efficiency in all test ranges (see Fig. 4). The integrated performance (Fig. 5) shows the flat-blade design with the optimized wet end components to be undoubtedly advantageous to the earlier tested designs of a better performance.

\section{Conclusion}

The obtained experimental data takes into account the specific nature of the HLMC effects on the pump wet end components and are recommended for use when justifying the reactor coolant pump designs for heavy liquid-metal cooled reactor plants.

low and medium power plants with lead and lead-bismuth coolants. VANT. Ser.: Fizika Yadernykh Reaktorov 2: 28-34. [in Russian]

- Beznosov AV, Dragunov YuG, Rachkov VI (2006) Heavy Liquid Metal Coolants in Nuclear Power. NGTU Publ., Nizhny Novgorod, 435 pp. [in Russian]

- Beznosov AV, Drozdov YuN, Antonenkov MA, Bokova TA, Lvov AV, Lemekhov VV (2014a) Experimental investigation of the wet end of an axial reactor coolant pump model for the heavy liquid metal cooled reactor facility. Vol. 2, 38-45. [in Russian]

- Beznosov AV, Lvov AV, Bokov PA, Bokova PA, Razin VA (2017) Experimental studies of the dependencies of the characteristics of axial pumps, pumped lead coolant, on the parameters of grids of profiles of impellers. Izvestiya vuzov. Yadernaya Energetika 1: 138144. https://doi.org/10.26583/npe.2017.1.13 [in Russian]

- Beznosov AV, Lvov AV, Bokova TA, Bokov PA, Lukichev NS (2019) Experimental studies of the dependence of the characteristics of axial pumps pumping lead coolant on the parameters of the 
straightening apparatus. Izvestiya vuzov. Yadernaya Energetika 2: 138-146. [in Russian]

- Beznosov AV, Lvov AV, Volkov NS, Lukichev NS, Menskaya KV, Bokov PA, Bokova TA, Novinsky EG, Marov AR (2019a) Experimental studies of the dependences of axial pumps pumping a lead heat carrier from the geometry of the exit plot of the blades of the work profiles of the worker wheel profiles. Trudy NGTU im. R.E. Alekseeva 1 (124): 76-82. [in Russian]

- Bokov PA (2015) Investigation of the Conditions for the Occurrence and Characteristics of Cavitation in Fast Neutron Heavy Liquid Metal Cooled Reactors. Cand. of Sc. (Eng.) diss. NGTU Publ., Nizhny Novgorod, 177 pp. [in Russian]

- Budov VM (1986) Pumps of Nuclear Power Plants. Energoatomizdat Publ., Moscow, 408 pp. [in Russian]

- Chechyotkin AV (1971) High Temperature Coolants. Energiya Publ., Moscow, 496 pp. [in Russian]
- Dragunov YuG, Lemekhov VV, Moiseyev AV, Smirnov VS (2015) Lead cooled fast neutron reactor (BREST). Problemy mashinostroyeniya i avtomatizatsii, 3: 97-103. [in Russian]

- Karelin VYa (1975) Cavitation Phenomena in Centrifugal and Axial Pumps. Mashinostroyeniye Publ., Moscow, 336 pp. [in Russian]

- Lomakin AA (1966) Centrifugal and Axial Pumps. Mashinostroyeniye Publ., Moscow - Leningrad, 364 pp. [in Russian]

- Mikhaylov AK, Malyushenko VV (1977) Paddle Pumps. Theory, Calculation and Design. Mashinostroyeniye Publ., Moscow, 288 pp. [in Russian]

- Papyr AN (1970) Water-Jet Propulsion of Small Vessels. Sudostroyeniye Publ., Leningrad, 256 pp. [in Russian]

- Rozhdestvenskiy VV (1977) Cavitation. Sudostroyeniye Publ., Leningrad, 247 pp. [in Russian] 